\title{
Clinical outcomes after delayed thyroid surgery in patients with papillary thyroid microcarcinoma
}

\author{
Min Ji Jeon', Won Gu Kim', Hyemi Kwon', Mijin Kim', Suyeon Park', Hye-Seon Oh', \\ Minkyu Han'2, Tae-Yon Sung ${ }^{3}$, Ki-Wook Chung ${ }^{3}$, Suck Joon Hong ${ }^{3}$, Tae Yong Kim', \\ Young Kee Shong ${ }^{1}$ and Won Bae Kim ${ }^{1}$
}

Departments of ${ }^{1}$ Internal Medicine, ${ }^{2}$ Clinical Epidemiology and Biostatistics and ${ }^{3}$ Surgery, Asan Medical Center, University of Ulsan College of Medicine, Seoul, Korea
Correspondence should be addressed to W G Kim

Email

wongukim@amc.seoul.kr

\begin{abstract}
Objective: Active surveillance is an option for patients with papillary thyroid microcarcinoma (PTMC). However, the long-term clinical outcomes after delayed surgery remain unclear. We compared the long-term clinical outcomes of PTMC patients according to the time interval between initial diagnosis and surgery.

Design and methods: In this individual risk factor-matched cohort study, PTMC patients were classified into three groups according to the delay period: $\leq 6$ months, $6-12$ months and $>12$ months. Patients were matched by age, sex, extent of surgery, initial tumor size as measured by ultrasonography (US), and by the presence of extrathyroidal extension, multifocal tumors and central cervical lymph node metastasis. We compared the dynamic risk stratification (DRS) and the development of structural persistent/recurrent disease of patients.

Results: A total of 2863 patients were assigned to three groups. Their mean age was 50 years, $81 \%$ were female and $66 \%$ underwent lobectomy. The mean tumor size at the initial US was $0.63 \mathrm{~cm}$. There were no significant differences in clinicopathological characteristics between groups after individual risk factor matching. Comparison of the DRS revealed no significant difference according to the delay period $(P=0.07)$. During the median 4.8 years of follow-up, there were no significant differences in the development of structural recurrent/persistent disease $(P=0.34)$ and disease-free survival $(P=0.25)$ between groups.

Conclusions: In PTMC patients, delayed surgery was not associated with higher risk of structural recurrent/persistent disease compared to immediate surgery. These findings support the notion that surgical treatment can be safely delayed in patients with PTMC under close monitoring.
\end{abstract}

\section{Introduction}

Recent studies have suggested more conservative management for small papillary thyroid carcinoma (PTC) including papillary thyroid microcarcinoma (PTMC), which is $\leq 1 \mathrm{~cm}$ in maximal tumor diameter. Generally, patients with PTMC have excellent prognosis and indolent disease course. Recently, an active surveillance approach was proposed as an alternative option for patients with PTMC, especially for those without any evidence
C 2017 European Society of Endocrinology Printed in Great Britain of invasion, metastases, or cytological or molecular aggressive features $(1,2)$. Low-risk PTMC patients can be closely monitored via serial neck ultrasonography (US) and physical examination with or without other imaging studies, and patients showing disease progression during follow-up or who choose to may proceed to surgery $(3,4$, $5,6)$. According to previous studies, active surveillance has favorable oncologic outcomes compared with immediate 
surgery with up to $15 \%$ of patients proceeding to surgery from active surveillance $(1,7)$.

Currently, there are no data regarding the differences in long-term clinical outcomes, such as the development of structural recurrent/persistent disease after initial therapy for PTMC, between immediate and delayed surgery. Because the timing of surgery might be affected by the clinicopathological characteristics of patients such as age, sex and the size or the position of the PTMC in neck US, which are also known risk factors for recurrence in PTMC $(1,8)$, the analysis should match the individual risk factors and compare the long-term clinical outcomes of PTMC patients with similar clinicopathological characteristics.

In this study, we aimed to evaluate the long-term clinical outcomes of PTMC patients according to the time interval from the initial diagnosis of PTMC to thyroid surgery (delay period). We classified the patients into three groups according to the delay period: within 6 months, 6-12 months and more than 12 months. We assessed the dynamic risk stratification (DRS) categories and disease-free survivals between the groups after individual matching of various clinicopathological characteristics of patients with PTMC.

\section{Subjects and methods}

\section{Study cohort}

This retrospective cohort study initially included 9885 consecutive patients with PTMC who underwent initial thyroid surgery between 1999 and 2013 at Asan Medical Center, Seoul, Korea. We excluded patients with incidental PTMCs, which were only identified postoperatively in thyroid specimens following thyroid surgery for presumed benign disease, and patients with lateral cervical lymph node (LN) metastases or distant metastases at initial diagnosis (clinical PTMCs) (9). We excluded patients without high-quality preoperative US images or adequate follow-up data. We also excluded patients with maximal tumor size greater than $1 \mathrm{~cm}$ at the initial US examination. A total of 6217 patients with PTMC were eligible for the unmatched PTMC cohort.

Patients were classified into three groups according to the time interval from the initial diagnosis to thyroid surgery (delay period). The time of initial diagnosis was defined as the time of initial identification of the suspicious thyroid nodules, which were finally confirmed as PTMC, by US examination. Patients who underwent immediate surgery within 6 months from the first diagnosis were classified into Group 1. Patients were classified into Group 2 when the delay period was 6-12 months and those who underwent delayed thyroid surgery after 12 months from the initial diagnosis were classified into Group 3. Group 3 had the smallest number of patients, so each group of patients was then individually matched in a 3:3:1 ratio (1227:1227:409 patients) according to their age, sex, the extent of surgery, maximum PTMC diameter at the initial neck US, the presence of extrathyroidal extension, multifocal tumors and central cervical LN metastasis at final pathology (Fig. 1). Differences within one year of age

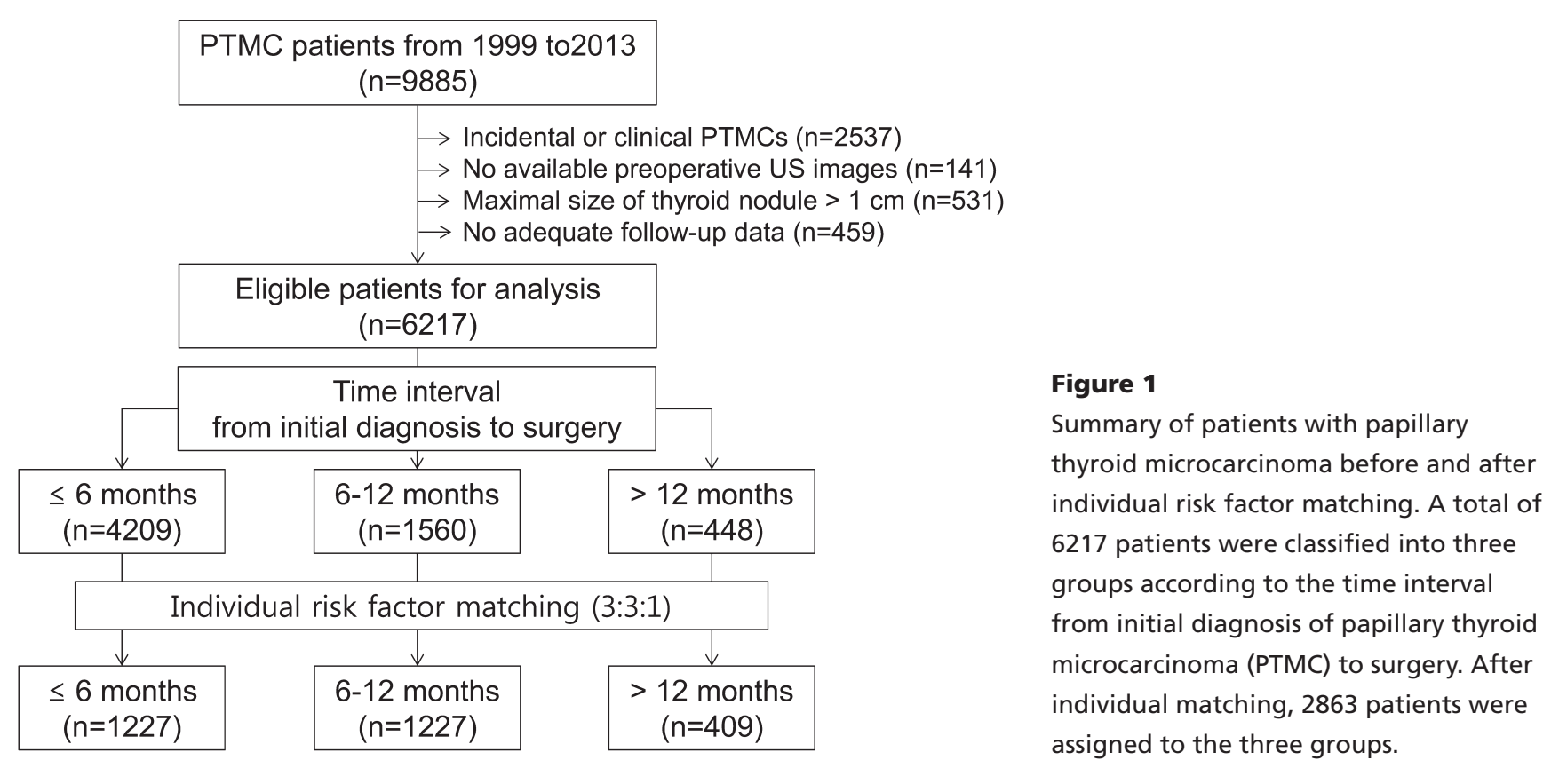

www.eje-online.org 
and within $0.1 \mathrm{~cm}$ of the primary tumor size were regarded as the same during individual risk factor matching. This study was approved by the institutional review board of Asan Medical Center.

\section{Management and follow-up protocol}

The follow-up protocol was shared at our center in order to maintain consistent management across the patients as previously reported (10). Routine preoperative neck US was performed in all patients. The timing and extent of surgery were individually determined depending on the patient's preference and surgeon's decision. Patients with bilateral multifocal PTMCs or PTMCs with indeterminate nodules in the contralateral lobe were treated by total thyroidectomy, whereas the other patients were treated by lobectomy. Prophylactic ipsilateral or bilateral central neck dissection was routinely performed for patients with PTMC. Radioactive iodine (RAI) remnant ablation after total thyroidectomy was conducted for patients with risk factors for recurrence (11).

After initial therapy, all patients were regularly followed up every 6-12 months with physical examinations, as well as the measurement of thyroid function, serum thyroglobulin ( $\mathrm{Tg})$, serum anti-Tg antibody (TgAb) and neck US. Diagnostic RAI whole-body scan (WBS) with measurement of the serum-stimulated $\mathrm{Tg}$ (sTg) levels was also performed 12-24 months after the initial therapy in patients who underwent total thyroidectomy and RAI remnant ablation as previously reported $(10,11)$.

\section{Definitions}

The DRS according to the response to initial therapy and the development of structural persistent/recurrent disease were the primary outcomes of this study.

The DRS categories were determined using classifications described in previous studies $(2,12,13$, $14,15)$ that employed serum $\mathrm{Tg}$ levels, serum $\mathrm{TgAb}$ levels and neck US with/without diagnostic WBS. Patients were classified into four groups according to their responses to initial therapy: excellent, indeterminate, biochemical incomplete and structural incomplete response.

Structural persistent/recurrent disease was defined as pathologically or cytologically proven recurrent or metastatic lesions and/or the appearance of metastatic lesions in other distant organs on imaging studies, with elevated Tg levels $(10,14)$. Disease-free survival (DFS) was defined as the time interval from the initial surgery to detection of structural persistent/recurrent disease.

\section{Statistics}

For individual matching, R version 3.0.1 was used. R Studio version 0.98.1091 and R libraries survival, car and gdata were used to analyze the matched data ( $\mathrm{R}$ Foundation for Statistical Computing, http://www.R-project.org/). Continuous variables were presented as means with standard deviation (s.D.) or medians with interquartile range (IQR). Categorical variables were presented as numbers with percentages. Chi-square or Fisher's exact tests were used to compare categorical variables, and oneway analysis of variance (ANOVA) was used to compare continuous variables.

DFS curves in the matched data set were constructed using the Kaplan-Meier method by GraphPad Prism version 5.01 (GraphPad Software). Log-rank tests were also performed to evaluate differences in the DFSs between groups. A Cox proportional hazard model was also used to evaluate the risk factors of structural recurrent/persistent disease. For the Cox proportional hazard model analysis, age and primary tumor size were categorized. An age of 45 years and a primary tumor size of $0.5 \mathrm{~cm}$ were the cut-off points for categorization (16). All $P$ values were two-sided, and $P<0.05$ was considered statistically significant.

\section{Results}

\section{Clinicopathological characteristics of patients with PTMC before individual risk factor matching}

The clinicopathological characteristics of 6217 patients before individual risk factor matching are shown in Table 1 (left panel). Mean age of the patients was 48.9 years, and 5142 (83\%) were female. The mean tumor size as measured by neck US at the diagnosis of PTMC was $0.65 \mathrm{~cm}$. Approximately half of the patients underwent lobectomy, and the others underwent total thyroidectomy. The mean primary tumor size at the final pathology was $0.60 \mathrm{~cm}$, and 1908 patients (31\%) had central cervical LN metastases. During a median of 4.8 years of follow-up (IQR: 3.6-6.5 years), 88 patients (1.4\%) were determined to have structural persistent/recurrent disease.

Patients were classified into three groups according to the delay period until the thyroid surgery. About $68 \%$ of patients underwent thyroid surgery within 6 months (Group 1), and 25\% underwent surgery between 6 and 12 months (Group 2). The delay period for thyroid surgery was longer than 12 months in 448 patients (7\%, Group 3). The median interval from initial diagnosis to surgery was 


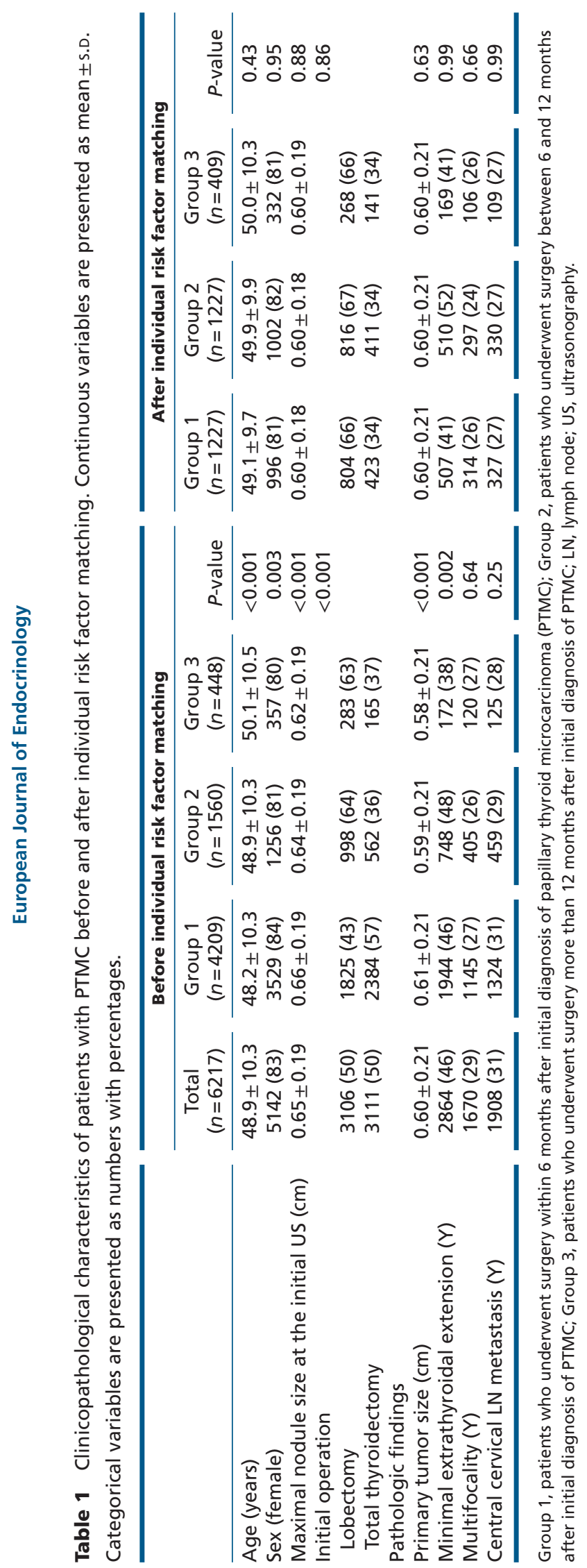

3 months (IQR: 1.6-4.4 months) in Group 1, 8 months (IQR: 6.7-9.4 months) in Group 2 and 17 months (IQR: 13.6-23.9 months) in Group 3. Patients who underwent delayed surgery tended to be older, male and have undergone lobectomy $(P<0.001, P=0.003$ and $P<0.001$, respectively). Furthermore, the size of the PTMC at their initial US and at final pathology tended to be smaller with the delay of surgery $(P<0.001$, and $P<0.001$, respectively) (Table 1).

\section{Clinicopathological characteristics of patients after individual risk factor matching}

After individual matching of age, sex, the size of PTMC measured at the initial US, the extent of surgery, the presence of extrathyroidal extension, multifocal tumors and cervical central LN metastasis at final pathology, 1227 patients each was assigned to Groups 1 and 2, and 409 patients were assigned to Group 3. In the matched data set, the mean age of patients was 50.0 years, and $81 \%$ of patients were female. The mean size of PTMCs measured in the initial US was $0.63 \mathrm{~cm}$, and about $66 \%$ of patients underwent lobectomy (Table 1, right panel). All preoperative and postoperative characteristics of patients with PTMCs were not significantly different between groups after individual matching.

The clinicopathological characteristics of patients with PTMC who were excluded after individual risk factor matching are shown in Supplementary Table 1 (see section on supplementary data given at the end of this article).

\section{Clinical outcomes of patients according to the thyroid surgery delay period}

We evaluated the DRS after initial therapy in patients with PTMC and observed no significant differences in the DRS categories between the groups $(P=0.07)$. Excellent responses were confirmed in 942 (77\%) patients in Group 1, 953 patients (78\%) in Group 2 and 303 patients (74\%) in Group 3 (Table 2). Only 20 patients (2\%) in Group 1, 7 patients $(0.6 \%)$ in Group 2 and two patients $(0.5 \%)$ in Group 3 presented with structural incomplete responses.

During follow-up, structural persistent/recurrent disease was detected in 19 patients (1.6\%) in Group 1, 13 patients $(1.1 \%)$ in Group 2 and three patients $(0.7 \%)$ in Group 3. The median duration from initial surgery to the detection of structural persistent/recurrent disease was 3.3 years (IQR: 1.3-5.0 years). There was no significant difference in the incidence of structural 
Table 2 Clinical outcomes of patients with PTMC after individual risk factor matching. Data are presented as $n$ (\%).

\begin{tabular}{lc}
\hline & $\begin{array}{c}\text { Group 1 } \\
(n=1227)\end{array}$ \\
\cline { 1 - 2 } $\begin{array}{lc}n \\
\text { Eynamic risk stratification }\end{array}$ & $942(77)$ \\
Indeterminate & $192(16)$ \\
Biochemical incomplete & $73(6)$ \\
Structural incomplete & $20(2)$ \\
Structural persistent/recurrent disease & $19(1.6)$ \\
Contralateral lobe & $5(26)$ \\
Postoperative bed/central cervical LN & $1(5)$ \\
Lateral cervical LN & $13(68)$ \\
\hline
\end{tabular}

LN, lymph node.

persistent/recurrent disease between the three groups $(P=0.34)$. The DFS curves also did not differ significantly between the groups (Fig. 2, $P=0.25$ ). When we subclassified the structural persistent/recurrent disease by the recurrence sites, there was no significant difference in the site of persistent/recurrent disease according to group $(P=0.38$, Table 2$)$.

Analysis of the risk factors for structural persistent/ recurrent disease of PTMC patients in the matched dataset revealed that male gender (hazard ratio $(\mathrm{HR})=2.43$, 95\% confidence interval (CI): 1.21-4.91, $P=0.013)$ and multifocal tumors (HR=2.45, 95\% CI: $1.24-4.83, P=0.010$ ) were independent risk factors (Table 3 ). The thyroid

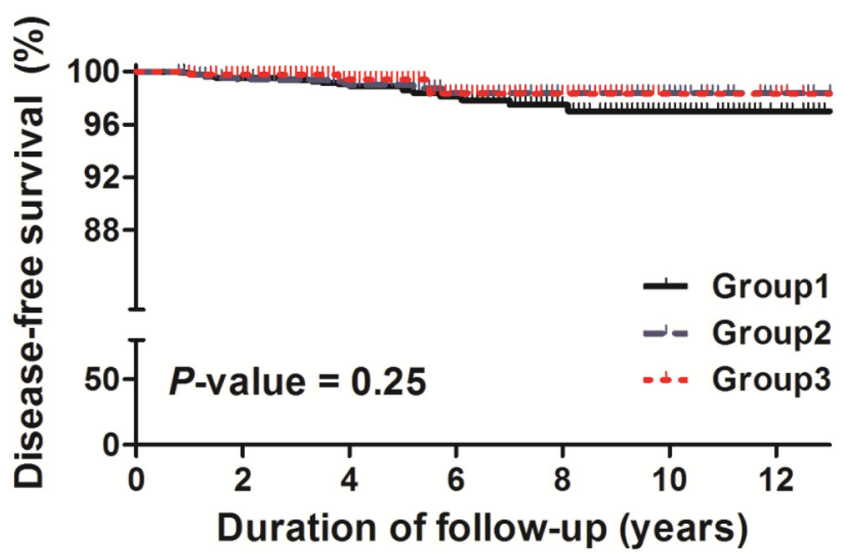

\section{Figure 2}

Disease-free survival curves of patients in three groups according to the thyroid surgery delay period after individual risk factor matching. Group 1, patients who underwent surgery within 6 months after initial diagnosis of papillary thyroid microcarcinoma (PTMC); Group 2, patients who underwent surgery 6-12 months after initial diagnosis of PTMC; Group 3, patients who underwent surgery more than 12 months after initial diagnosis of PTMC. $P$-value was determined by the log-rank test.

\begin{tabular}{|c|c|c|}
\hline $\begin{array}{c}\text { Group 2 } \\
(n=1227)\end{array}$ & $\begin{array}{c}\text { Group } 3 \\
(n=409)\end{array}$ & $P$-value \\
\hline & & 0.07 \\
\hline $953(78)$ & 303 (74) & \\
\hline 189 (15) & 78 (19) & \\
\hline $78(6)$ & $26(7)$ & \\
\hline $7(0.6)$ & $2(0.5)$ & \\
\hline $13(1.1)$ & $3(0.7)$ & 0.34 \\
\hline $5(38)$ & $1(33)$ & 0.38 \\
\hline $2(15)$ & $1(33)$ & \\
\hline $6(46)$ & $1(33)$ & \\
\hline
\end{tabular}

surgery delay period was not associated with the risk of structural persistent/recurrent disease in PTMC patients.

\section{Discussion}

In this study, we evaluated the impacts of delayed thyroid surgery on the long-term clinical outcomes of patients with PTMC. We used a matched data set by individually matching patient age, sex, extent of surgery, size of PTMC measured at the initial US, the presence of extrathyroidal extension, multifocal tumors and cervical central LN metastasis at final pathology in order to minimize other confounding factors associated with long-term clinical outcomes of PTMC. The thyroid surgery delay period was classified into three groups: within 6 months, between 6 and 12 months and more than 12 months. There were no significant differences in the DRS categories and the development of structural recurrent/persistent disease in PTMC patients between the groups. These findings suggest that delayed surgery may not affect the long-term clinical outcomes of low-risk patients with PTMC.

Active surveillance is a conservative observational approach and gained attention when it was first reported in PTMC $(1,17)$. However, this approach was only validated in Japan in retrospective observational studies, and there are concerns of disease progression during active surveillance, especially in young patients $(1,18)$. Previous studies from Japan reported that disease progression during active surveillance could be treated successfully with thyroid surgery (7), but the long-term disease outcome was not elucidated. In the present study, delayed surgery (median 17 months after initial diagnosis) was not associated with an increased risk for structural recurrent/ persistent disease compared to immediate surgery. There were also no differences in the DRS categories after initial therapy according to the delay period from diagnosis 
Table 3 Univariate and multivariate analyses of risk factors for structural recurrent/persistent disease of PTMC.

\begin{tabular}{l}
\hline Age ( $\geq 45$ years) \\
Sex (male) \\
Extent of operation $(T T)$ \\
Primary tumor size $(>0.5 \mathrm{~cm}$ ) \\
Minimal extrathyroidal extension $(\mathrm{Y})$ \\
Multifocality $(\mathrm{Y})$ \\
Cervical central LN metastasis $(\mathrm{Y})$ \\
Delayed period to surgery \\
Group 1 \\
Group 2 \\
Group 3
\end{tabular}

\begin{tabular}{c}
\hline HR \\
\hline 0.84 \\
2.54 \\
1.12 \\
2.24 \\
1.59 \\
3.01 \\
2.18 \\
\\
Ref. \\
0.76 \\
0.59 \\
\hline
\end{tabular}

\begin{tabular}{c}
\hline $\mathbf{9 5} \% \mathbf{C l}$ \\
\hline $0.42-1.70$ \\
$1.26-5.11$ \\
$0.57-2.22$ \\
$1.08-4.67$ \\
$0.82-3.09$ \\
$1.55-5.85$ \\
$1.12-4.23$
\end{tabular}

\begin{tabular}{c}
\hline P-value \\
\hline 0.63 \\
$<0.001$ \\
0.74 \\
0.031 \\
0.17 \\
0.001 \\
0.022
\end{tabular}

\begin{tabular}{c}
\hline HR \\
\hline 2.43 \\
1.76
\end{tabular}

$\frac{95 \% \mathrm{Cl}}{1.21-4.91}$

P-value

2.45

$0.83-3.75$

0.013

$1.12-4.23$

1.77

$1.24-4.83$

0.14

$0.90-3.49$

0.010

0.10

$\mathrm{Cl}$, confidence interval; HR, hazard ratio; Ref, reference; TT, total thyroidectomy; $\mathrm{Y}$, yes.

to thyroid surgery. Even though the patients who had delayed surgery in this study were not those who showed disease progression during active surveillance, our findings suggest that delayed surgery 1-2 years after the initial diagnosis might not be unfavorable and provides indirect evidence for the safe application of active surveillance for low-risk PTMCs.

Before individual risk factor matching, patients in Group 3, who underwent surgery 12 months after initial diagnosis, were likely to be older, male and have smaller tumors compared to those in Groups 1 and 2. These are well-known factors associated with the recurrence of PTMCs $(8,11,16)$ and can affect a surgeon's decision to perform surgery. Therefore, we evaluated the clinical outcomes after individual matching of patient age, sex, primary tumor size as well as the extent of surgery. Furthermore, the number or position of suspicious nodules at initial US might affect the decision to perform surgery; therefore, we matched all other pathological variables such as the presence of extrathyroidal extension, multifocal tumors and central cervical LN metastasis at final pathology in this study. In these matched datasets, only male gender and multifocal tumors were independent risk factors of the structural persistent/recurrent disease in patients with PTMC, and delay period was not associated with the risk of structural persistent/recurrent disease in PTMC patients.

This study has limitations due to its retrospective design that included patients from a single tertiary referral center. We could not evaluate the exact reasons for delayed or immediate surgery based on our review of the medical records. We also could not evaluate how many patients in the Group 3 had delayed surgery due to disease progression during observation. Thus, the result of this study could not give adequate information about the role of active surveillance in PTMC. However, our study has several advantages, including its long-term evaluation of the clinical outcomes of patients who underwent delayed thyroid surgery compared with balanced control groups in low-risk patients with PTMC.

In conclusion, after individual risk factor matching, delayed surgery for patients with PTMC was not associated with increased risk for structural recurrent/persistent disease compared to immediate surgery. These findings support that the surgical treatment can be safely delayed in patients with PTMC under close monitoring.

Supplementary data

This is linked to the online version of the paper at http://dx.doi.org/10.1530/ EJE-17-0160.

Declaration of interest

The authors declare that there is no conflict of interest that could be perceived as prejudicing the impartiality of this study.

\section{Funding}

This study was supported by a grant from the Korean Health Technology R\&D project, Ministry of Health \& Welfare, Republic of Korea (HC15C3372), and a grant (No. 2017-582) from the Asan Institute for Life Sciences, Asan Medical Center, Seoul, Korea.

\section{References}

1 Ito Y, Miyauchi A, Kihara M, Higashiyama T, Kobayashi K \& Miya A. Patient age is significantly related to the progression of papillary microcarcinoma of the thyroid under observation. Thyroid 201424 27-34. (doi:10.1089/thy.2013.0367)

2 Haugen BR, Alexander EK, Bible KC, Doherty GM, Mandel SJ, Nikiforov YE, Pacini F, Randolph GW, Sawka AM, Schlumberger M et al. 2015 American Thyroid Association Management Guidelines for adult patients with thyroid nodules and differentiated thyroid cancer: the American Thyroid Association Guidelines task force on thyroid nodules and differentiated thyroid cancer. Thyroid 201626 1-133. (doi:10.1089/thy.2015.0020)

3 Haser GC, Tuttle RM, Su HK, Alon EE, Bergman D, Bernet V, Brett E, Cobin R, Dewey EH, Doherty G et al. Active surveillance for papillary thyroid microcarcinoma: new challenges and opportunities for the 
health care system. Endocrine Practices 201622 602-611. (doi:10.4158/ EP151065.RA)

4 Tufano RP, Shindo M \& Shaha AR. New recommendations for extent of thyroidectomy and active surveillance for the treatment of differentiated thyroid cancer. JAMA Otolaryngology: Head and Neck Surgery 2016142 625-626. (doi:10.1001/jamaoto.2016.0980)

5 Brito JP, Ito Y, Miyauchi A \& Tuttle RM. A clinical framework to facilitate risk stratification when considering an active surveillance alternative to immediate biopsy and surgery in papillary microcarcinoma. Thyroid 201626 144-149. (doi:10.1089/thy.2015.0178)

6 Yi KH. The revised 2016 Korean Thyroid Association Guidelines for thyroid nodules and cancers: differences from the 2015 American Thyroid Association Guidelines. Endocrinology and Metabolism 201631 373-378. (doi:10.3803/EnM.2016.31.3.373)

7 Oda H, Miyauchi A, Ito Y, Yoshioka K, Nakayama A, Sasai H, Masuoka H, Yabuta T, Fukushima M, Higashiyama T et al. Incidences of unfavorable events in the management of low-risk papillary microcarcinoma of the thyroid by active surveillance versus immediate surgery. Thyroid 201626 150-155. (doi:10.1089/ thy.2015.0313)

8 Pisanu A, Saba A, Podda M, Reccia I \& Uccheddu A. Nodal metastasis and recurrence in papillary thyroid microcarcinoma. Endocrine 2015 48 575-581. (doi:10.1007/s12020-014-0350-7)

9 Mehanna H, Al-Maqbili T, Carter B, Martin E, Campain N, Watkinson J, McCabe C, Boelaert K \& Franklyn JA. Differences in the recurrence and mortality outcomes rates of incidental and nonincidental papillary thyroid microcarcinoma: a systematic review and metaanalysis of 21329 person-years of follow-up. Journal of Clinical Endocrinology and Metabolism 201499 2834-2843. (doi:10.1210/ jc.2013-2118)

10 Jeon MJ, Kim WG, Choi YM, Kwon H, Song DE, Lee YM, Sung TY, Yoon JH, Hong SJ, Baek JH et al. Recent changes in the clinical outcome of papillary thyroid carcinoma with cervical lymph node metastasis. Journal of Clinical Endocrinology and Metabolism 2015100 3470-3477. (doi:10.1210/JC.2015-2084)

11 Jeon MJ, Kim WG, Choi YM, Kwon H, Lee YM, Sung TY, Yoon JH, Chung KW, Hong SJ, Kim TY et al. Features predictive of distant metastasis in papillary thyroid microcarcinomas. Thyroid 201626 161-168. (doi:10.1089/thy.2015.0375)

12 Park S, Kim WG, Song E, Oh HS, Kim M, Kwon H, Jeon MJ, Kim TY, Shong YK \& Kim WB. Dynamic risk stratification for predicting recurrence in patients with differentiated thyroid cancer treated without radioactive iodine remnant ablation therapy. Thyroid $2017 \mathbf{2 7}$ 524-530. (doi:10.1089/thy.2016.0477)

13 Momesso DP, Vaisman F, Yang SP, Bulzico DA, Corbo R, Vaisman M \& Tuttle RM. Dynamic risk stratification in patients with differentiated thyroid cancer treated without radioactive iodine. Journal of Clinical Endocrinology and Metabolism 2016101 2692-2700. (doi:10.1210/ jc.2015-4290)

14 Jeon MJ, Kim WG, Park WR, Han JM, Kim TY, Song DE, Chung KW, Ryu JS, Hong SJ, Shong YK et al. Modified dynamic risk stratification for predicting recurrence using the response to initial therapy in patients with differentiated thyroid carcinoma. European Journal of Endocrinology 2014170 23-30. (doi:10.1530/EJE-13-0524)

15 Tuttle RM, Tala H, Shah J, Leboeuf R, Ghossein R, Gonen M, Brokhin M, Omry G, Fagin JA \& Shaha A. Estimating risk of recurrence in differentiated thyroid cancer after total thyroidectomy and radioactive iodine remnant ablation: using response to therapy variables to modify the initial risk estimates predicted by the new American Thyroid Association staging system. Thyroid 201020 1341-1349. (doi:10.1089/thy.2010.0178)

16 Siddiqui S, White MG, Antic T, Grogan RH, Angelos P, Kaplan EL $\&$ Cipriani NA. Clinical and pathologic predictors of lymph node metastasis and recurrence in papillary thyroid microcarcinoma. Thyroid 201626 807-815. (doi:10.1089/thy.2015.0429)

17 Sugitani I, Toda K, Yamada K, Yamamoto N, Ikenaga M \& Fujimoto Y. Three distinctly different kinds of papillary thyroid microcarcinoma should be recognized: our treatment strategies and outcomes. World Journal of Surgery 201034 1222-1231. (doi:10.1007/s00268-0090359-x)

18 Kandil E, Noureldine SI \& Tufano RP. Thyroidectomy vs active surveillance for subcentimeter papillary thyroid cancers - the cost conundrum. JAMA Otolaryngology: Head and Neck Surgery 2016142 9-10. (doi:10.1001/jamaoto.2015.2852)

Received 24 February 2017

Revised version received 12 April 2017

Accepted 21 April 2017 